\title{
Localization of Facial Features Using Viola- Jones and Canny Method
}

\author{
Ranjana Sikarwar, Priyanka Nema \\ Department of CSE, St. Aloysius Institute of Technology, Jabalpur \\ Department of CSE, St. Aloysius Institute of Technology, Jabalpur
}

\begin{abstract}
Face detection plays a vital role in the field of image processing. The primary aim of the proposed face detection system is to find out if there is any face in an image and then localize the facial features in a human face. A hybrid approach is used for face detection in this paper which is a combination of neural networks used to detect faces using Multilayer feed forward networks and other using viola-Jones face detection method followed by canny edge detection method. Simulation results are taken in parameters of Hit Rate, Miss Rate, Accuracy, Run time.
\end{abstract}

Keywords: Canny, FAR, Hit Rate

\section{Introduction}

Face localization, detection and then identification or confirmation has been a testing errand because of various components. Face is so non-unbending and has so many variations that no system can adapt to all these varieties. That is the reason why in spite a large number of algorithms and techniques a robust system is still far from real implementation. Some of the variables are represented here.

Pose: Faces in images may have distinctive postures. The position of the face may differ while catching picture by cam or other gadget. Because of this variety face detection get to be troublesome as nose and eyes make distinctive edges.

Light: It is an alternate enormous test to identify precisely faces. Illumination problem have a bigger effect with the same face as contrast with distinction inside diverse faces while looking at appearances.

Occulusion: Faces in images some times are blocked with different objects. Beard, mustaches, optical lenses and different sorts of object make it test to discover exact face behind them.

Facial Expressions: Facial expressions of an individual while imaging have a wide effect for face detection process. Same individual may have diverse expressions at distinctive times.

Image Conditions: Image conditions likewise assume an imperative part amid face detection process. The cam lighting, background, distance between camera and person, intensity and resolution of the image are essential components. Qualities of Image catching devices influence the conditions of images and faces too.

Face Size: Variation in face sizes additionally make hard to mechanize a framework for face detection and recognition.

\section{Literature Review}

Feris [1] states that each feature is found within 3 pixels of the landmarks points in $\sim 95 \%$ of cases for eye corners and nostrils and in $\sim 88 \%$ of cases for mouth corners. The test set is a subset of the Feret database [80], where the faces have an approximate inter-ocular separation of 50-60 pixels. Here, 3 pixels represents 5$6 \%$ of the interocular separation, so the GWN method is accurate, but not always reliable and is only tested on a relatively clean data set. The method is also rather slow, so is unsuitable for real-time applications.

Jesorsky et al. [2] use the $\mathrm{S}^{\prime \prime}$ obel filter to detect edges in the image and then match strong edges to a face edge model using the Hausdorff distance [81]. This initial face localization method is then extended by searching with a smaller model to refine the eye locations, as shown in Figure 2.3. The location of each pupil is further refined using a MLP based detector for each eye.

Reinders et al. [3] describe a system to detect the right eye using neural networks and orientation maps. Four eye features are detected, namely the inner and outer corners of the eye and the upper and lower eyelids. The configuration of strong responses from each of the 4 neural networks is used to determined the likelihood of a region being the true location of the right eye. The system detects $96 \%$ of right eyes within 2 pixels, on faces with an inter-ocular separation of $20 \pm 2$ pixels. However, a good initialisation is required, for the eye finder. In fact, Reinders et al. use video and initialise the search from the previous frame, which limits the search region for the eye detector. It is unclear how well this system would work if the eye search was less constrained.

Shakunaga et al. [4] describe a system which combines the Eigen-template approach of Turk and Pentland [84] and a 3D model of the human face. Eight facial features are used; namely the eyes, eye brows, ears, mouth and nose. A common Eigen-space is built for each feature with a training set of left, right and 
frontal views. These detectors scan the image to produce a set of feature candidates that also indicate a direction (i.e. left/right/frontal). The feature candidates are combined into groups to form face candidates, subject to geometric constraints. The approximate pose of the face is estimated and a 3D shape model used to predict the location of missing features. A refined search for missing features then takes place. The final evaluation function is a weighted combination of the sum of feature responses, the fit with the 3D shape model and a penalty for missing features.

Shakunaga report finding $\sim 98 \%$ of the internal facial features and $\sim 87 \%$ of the ears testing on frontal face images and a few percent worse results using a test set with the subject at $30 \mathrm{o}$ to the camera. However, they do not specify the accuracy required to define a correctly detected feature and only test on their own data. They also appear to only test performance at discrete angles i.e. $-30 / 0 /+30$ degrees and do not comment on intermediate angles. The speed of the system is not commented upon.

\section{Proposed Methodology}

The hybrid approach used is demonstrated through the block diagram in fig1.The proposed algorithm used in this paper is used to detect upright frontal views of human faces in images accompanied by the extraction of facial features using canny method. Multilayer feed forward neural network is trained to distinguish faces and non- faces. Viola-Jones method detects faces using Haar-like features.

\subsection{Multilayer Feed forward network as a classifier}

In our detection, we used 500 images for faces and 100 for non faces for our database in different scale. Facial images also consist of animal images and non- facial images are taken of different objects The test images in a dataset are shown in figure below.
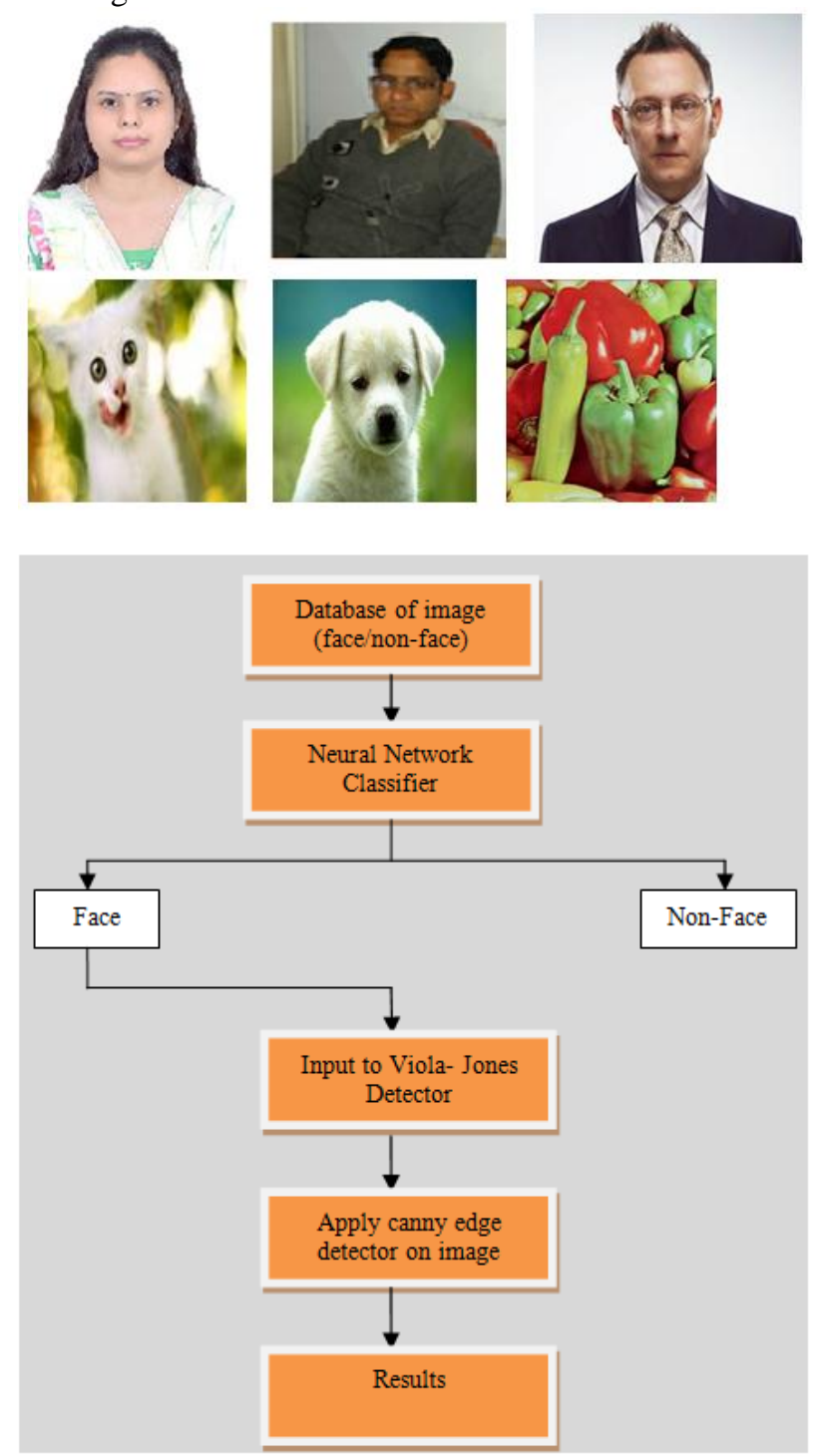

Fig .1: Block Diagram of Face Detection Approach 
Feed-forward networks: All associations point in one heading (from the input toward the output layer). It has the associated qualities:

1. Perceptrons are organized in layers, with the primary layer taking in inputs and the last layer yielding the outputs. The intermediate layers have no association with the outside world, and consequently are called hidden layers shown in fig. 2.

2. Each perceptron in one layer is associated with each perceptron on the following layer. Subsequently data is always "feed forward" starting with one layer then onto the next, and this clarifies why these systems are called feed-forward networks.

3. There is no association among perceptrons in the same layer.

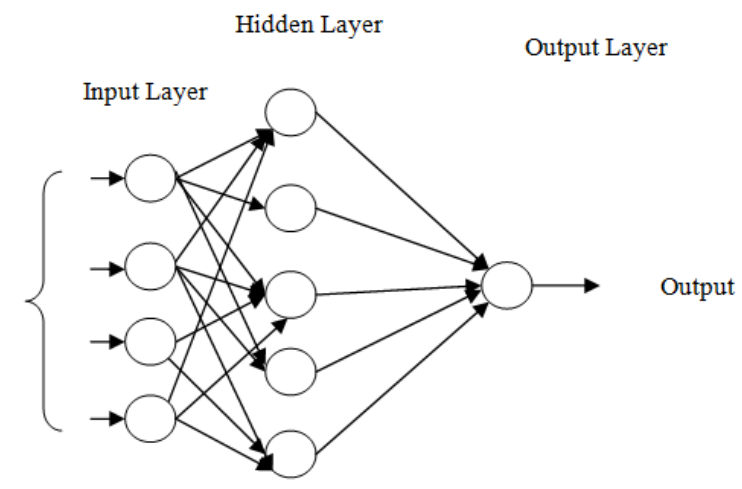

Figure 2. Feed-forward network [70]

Scale Gradient Conjugate (SCG) a type of training algorithm which has shown a good level of performance in face classification. As seen from the experimental results SCG converges and reduces the error with minimum iterations as compared to other training algorithms. Graph 1 illustrates the performance of neural network.

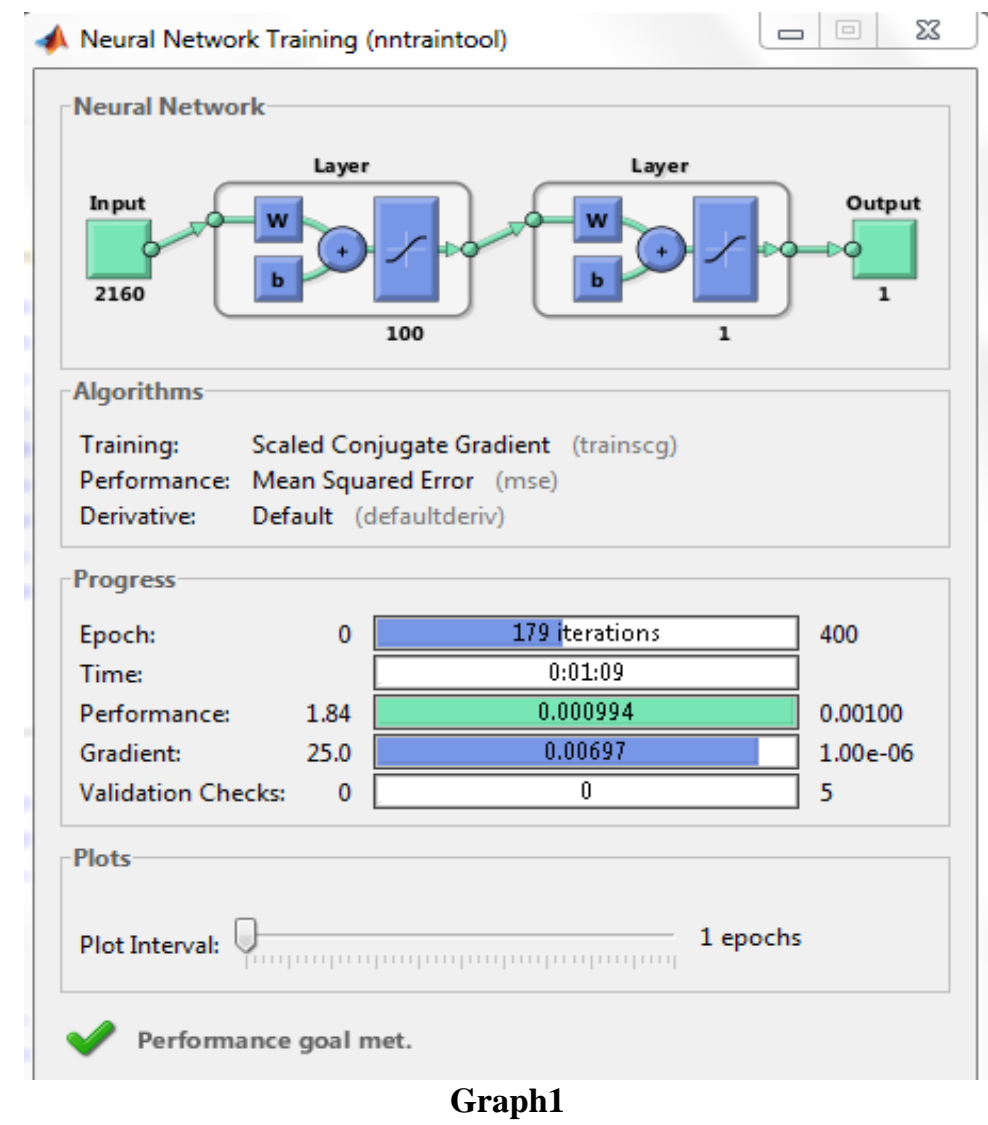

The output of this classifier is then used as an input to viola- Jones algorithm for face detection and feature extraction. 


\subsection{Viola- Jones Face detector}

The Viola- Jones face detector creates bounding boxes on face and then facial features. The cascade object detector uses the Viola - Jones algorithm to identify human faces, noses, eyes or mouth as shown in figure 3.

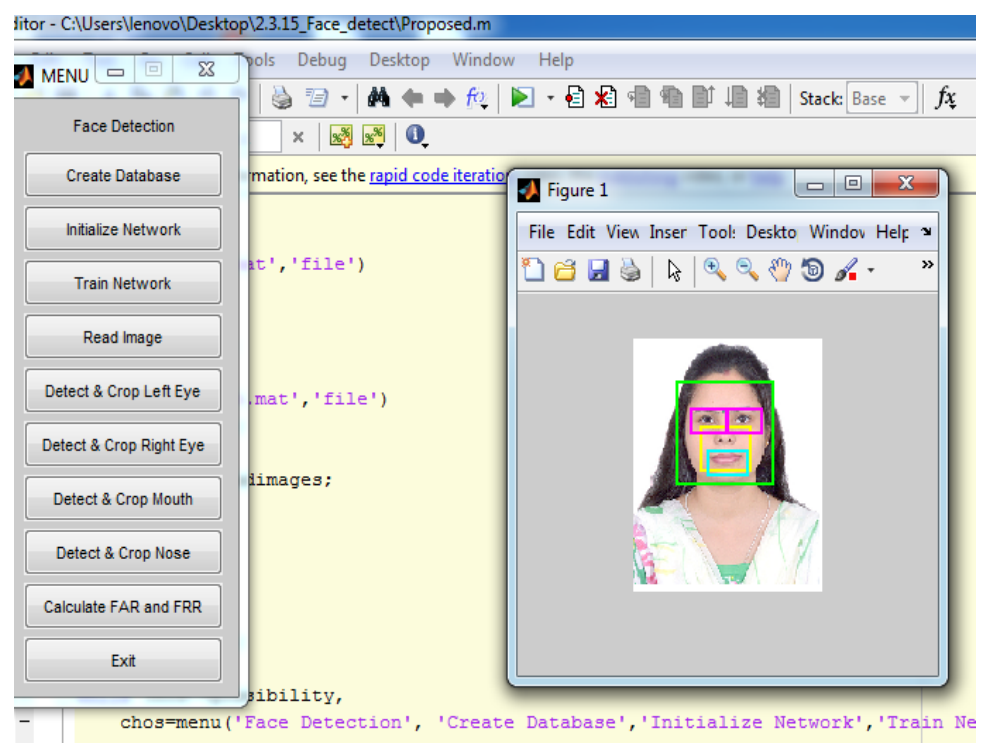

Fig. 3 Viola- Jones Face detector

detector $=$ vision. CascadeObjectDetector creates a System object, detector, that is used to detect objects using Viola-Jones algorithm.

\subsection{Edge detection using canny method}

The Canny Edge Detector is a standout amongst the most ordinarily utilized image processing tools, identifying edges in an exceptionally powerful way. It is a multi-step process, which can be actualized on the GPU as a sequence of filters. "Canny Edge Detection" goes over the methodology of making the creating the canny edge detection. The algorithm identifies edges taking into account the pixel intensity values within a certain threshold as shown in figure 3 below [5].

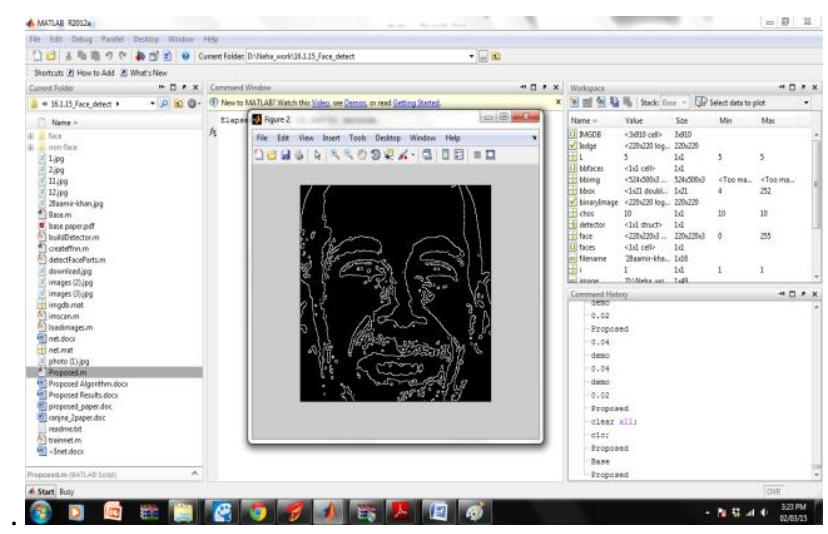

Figure 4. Image after canny filter

\section{Result Analysis}

We have used MATLAB R2012A to simulate the experiment and find out the results. Results are calculated in terms of Num of Hit, Num of missed faces, False Acceptance rate and False Rejection Rate finally. Firstly we have taken images one by one and executed through the system whether they are accepted by the system or rejected by the system. The False positive rate means number of correct faces missed and false negative rate means number of incorrect faces accepted by the system and False Acceptance Rate means false faces detected correctly and False Rejection Rate means number of correct faces rejected respectively. On a data set of 500 images containing both face and non-face images we have found the results as shown in table 1 and graph 2 on an average. Also in graph 2 we have compared our results with that of a system which uses Neural network with Adaboost(base system)[20] and results in high false positive rate. 
The accuracy is calculated by the equation below [18].

Also overall result of the proposed system is as shown in fig. below

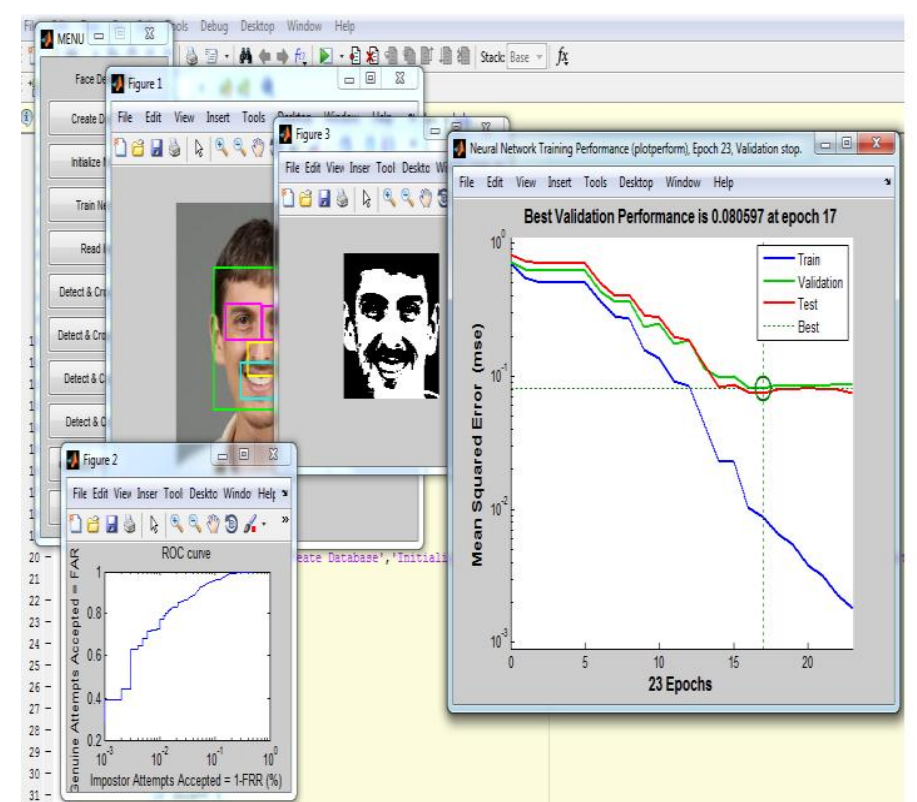

Figure.5 Proposed System performance graph

Accuracy $($ in $\%)=100$-(false positive rate+ false negative rate)

Table 1. Computation of Face Detection Performance

\begin{tabular}{|l|l|l|l|l|l|}
\hline Images & Num of faces & Hit Rate & Miss Rate & Run time(sec) & Accuracy \\
\hline Image1.jpg & 1 & 3.4997 & 1.8836 & 14.343587 & 94.6167 \\
\hline Image2.jpg & 1 & 3.3583 & 1.7077 & 16.265462 & 94.9340 \\
\hline Image3.jpg & 1 & 3.3583 & 1.7077 & 28.336507 & 94.9340 \\
\hline Image4.jpg & 1 & 3.3583 & 1.7077 & 15.52813 & 94.9340 \\
\hline Image5.jpg & 1 & 3.3583 & 1.7077 & 20.052301 & 94.9340 \\
\hline
\end{tabular}

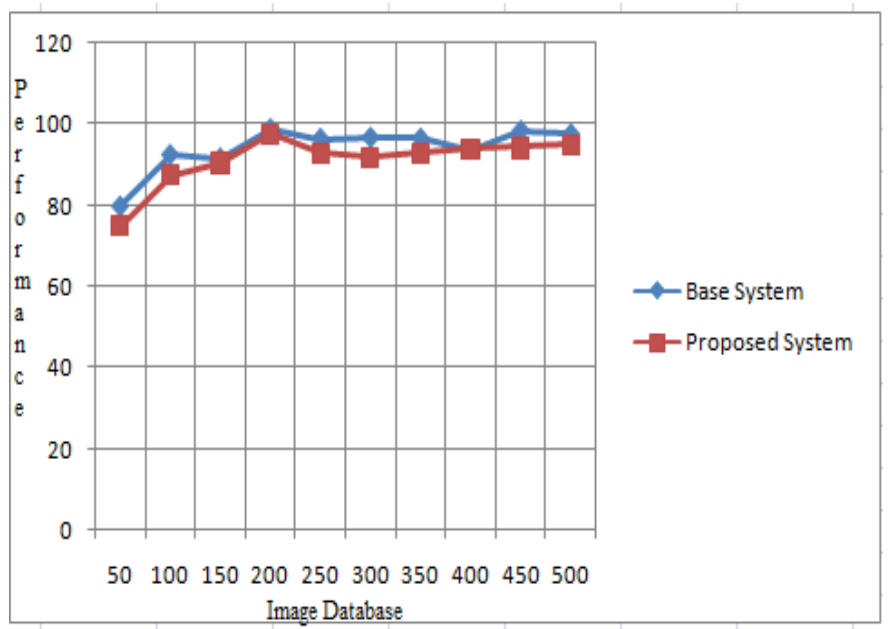

Figure.6 Performance for 500 images

\section{Conclusion}

At an early stage of the experiment we used only few numbers of 2D to test the working capability of the procedure and to decrease the training time of neural networks. Later on we used a larger number of test images compromising with the execution time but producing accurate results than the one used with Adaboost algorithm for face detection. Also using Adaboost produces high false positive rates. Also we can improve the image quality by preprocessing it through histogram equalization before testing, it is expected to produce much better results. In future this algorithm can be applied to detect human faces at multi-view points as well; currently it is restricted to upright frontal views of the face only. 


\section{References}

[1]. R. S. Feris, J. Gemmell, K. Toyama, and V.Kr̈uger. Hierarchical wavelet networks for facial feature localization. In 5th International Conference on Auto-matic Face and Gesture Recognition 2002, Washington, USA, Washington D.C., USA, May 2002 .

[2]. Jesorsky, K. J. Kirchberg, and R. W. Frischholz. Robust face detection using the hausdorff distance. In 3rd International Conference on Audio- and Video-Based Biometric Person Authentication 2001, 2001

[3]. M. Reinders, R. Koch, and J. Gerbrands. Locating facial features in image sequences using neural networks. In 2 nd International Conference on Automatic Face and Gesture Recognition 1996, pages 230-236, 1996.

[4]. T. Shakunaga, K. Ogawa, and S. Oki. Integration of eigentemplate and structured matching for autonomous facial feature detection. In 3rd International Conference on Automatic Face and Gesture Recognition 1998, 1998.

[5]. William, K. Pratt, "Digital Image Processing", Fourth Edition, A John Wiley \& Sons Inc. Publication, pp.465-529, 2007.

[6]. Leung, T.K.; Burl, M.C.; Perona, P., "Finding faces in cluttered scenes using random labeled graph matching",Computer Vision, 1995. Proceedings., Fifth International Conference on 20-23 June 1995 Page(s):637 - 644.

[7]. Sung,K.-K, Poggio T, "Example based learning for view based human face detection" Pattern Analysis and Machine Intelligence, IEEE transactions on, volume 0,Issue 1 Jan 1998, Pages( $39-51)$.

[8]. H. Schneiderman and T.Kanade. Probablistic modelling of local appearance and spatial relationships for object recognition. In Computer Vision and Pattern Recognition Conference 1998, 1998.

[9]. Rowley, HA:, Baluja, S:, Kanade, T:, "Neural Network-Based face Detection"Computer Vision and Pattern Recognition, 1996 Proceedings CVPR96, 1996 IEEE Computer Society Conference on, 18-20 June 1996, pages 203-208.

[10]. Rowley, HA:, Baluja, S:, Kanade, T:, "Rotation invariant Neural Network-Based face Detection"Computer Vision and Pattern Recognition, 1998. Proceedings. 1998 IEEE Computer Society Conference on 23-25 June 1998 Page(s):963 - 963.

[11]. http://homepages.inf.ed.ac.uk/rbf/CVonline/low/edges/canny.htm S Price, "Edges: The Canny Edge Detector", July 4, 1996.

[12]. Cristinacce, D., Cootes, T., and Scott, I. (2004). A multistage approach to facial feature detection. In 15th British Machine Vision Conference, 231-240.

[13]. X. Wang and X. Tang, "Face Photo-Sketch Synthesis and Recognition," IEEE Transactions on Pattern Analysis and Machine Intelligence (PAMI), Vol. 31, 2009.

[14]. Extract of Facial Feature Point, IJCSNS International Journal of Computer Science and Network Security, VOL.9 No.1, January 2009

[15]. Neural Network Based Approach for Face Detection cum Face Recognition, World Academy of Science, Engineering and Technology.

[16]. Face detection, Inseong Kim, Joon Hyung Shim, and Jinkyu Yang.

[17]. Robust Face Detection Method Based on Skin Color and Edges, J Inf Process Syst, Vol.9, No.1, March 2013. [18] Detecting Boundaries for Segmentation and Recognition.

[18]. Phil Brimblecombe (2002) "Face Detection using Neural Networks", H615 - Meng Electronic Engineering, School of Electronics and Physical Sciences, URN: 1046063.

[19]. Lixin Fan kah-kay sung, "Model -based varying pose face detection and facial feature registration in color images" 2002.

[20]. Zulhadi Zakaria"Face Detection Using Combination of Neural Network and Adaboost" Intelligent Biometric Group School of Electrical and Electronics Engineering Universiti Sains Malaysia.

[21]. S.P.Khandait, Dr. R.C.Thool, "Hybrid Skin Detection Algorithm for Face localization in facial Expression Recognition", Proceedings of international conference on Advance computing conference-09 (IACC- 09), Patiala, Punjab, India, 6-7 March'09.

[22]. Henry A. Rowley, Shumeet Baluja, and Takeo Kanade. Neural network based face detection. IEEE Trans. Pattern Anal. Mach. Intell., 20(1):23-38, 1998.

[23]. H. A. Rowley, S. Baluja, and T. Kanade. Human face detection in visual scenes. In D. S. Touretzky, M. C. Mozer, and M. E. Hasselmo, editors, Advances in Neural Information Processing Systems, volume 8, pages 875-881. The MIT Press, 1996.

[24]. Kotropoulos, C. Pitas, I, "Rule-based face detection in frontal views" Acoustics, Speech, and Signal Processing, 1997. ICASSP-97., 1997 IEEE International Conference on Volume 4, 21-24 April 1997 Page(s):2537 - 2540 vol.4. 